\title{
Optimal photon localization
}

\author{
J. Mourad \\ Laboratoire de Physique Théorique et Hautes Energies* \\ Bat. 211, Université Paris-Sud, 91405 Orsay, France.
}

\begin{abstract}
We show that there is only one operator having some minimal properties enabling it to be a one photon position operator. These properties are stated, and the solution is shown to be the photon position operator proposed by Pryce. This operator has non-commuting components. Nevertheless, it is shown that one can find states localized with an arbitrary precision.
\end{abstract}

\footnotetext{
* Laboratoire associé au CNRS
} 


\section{Introduction}

The experimental possibility of preparing one photon states greatly motivates the question of the photon localizability [1]. The question we wish to answer is the following: suppose we know the wave function of the photon, can we calculate the probability of finding it somewhere in space at a particular time ?

Different answers have been given to this question. Newton and Wigner [2] and later on Wightman [3] argued that the answer is negative, while Jauch, Piron [4] and Amrein [5] gave a positive answer at the expense of changing the axioms of localizability. ( They essentially dropped the requirement that the probability of finding a particle in a space region $R$ is the sum of the probabilities of finding it in the disjoint sub-regions $R_{i}$, where $\bigcup_{i} R_{i}=R$ ). The axioms of Newton and Wigner were for exactly localized sates, they therfore assumed that if no such states exist the particle is not localizable. In other words, they proved that no position operator with commuting components exists for the photon.

In this letter we drop the assumption of commuting components and retain the other Newton-Wigner axioms. We show, in section 2, that there exists an operator obeying these axioms and that this operator is unique. This operator was found for the first time by Pryce while looking for the analogue of the centre of mass for relativistic wave equations [6,7]. We will call it the Pryce operator.

Next we show, in section 3, that although exactly localized states do not exist, one can find states that are localized within an arbitrary small region of non vanishing volume. We argue that the Pryce operator can describe the position of the photon and gives means to answer the question we asked at the beginning.

\section{The position operator}

The photon is described by two irreducible representations of the Poincaré group [8], one for each helicity state. The minimal description of these representations is by means of a two-component wave function. However, this description is singular, the generators of the Poincaré group having Dirac-like monopole singularities, and the wave functions being elements of an associated Hilbert space over a U(1) -fiber bundle [9]. Another description, equivalent to the previous one is obtained by taking the zero mass limit of the massive representation of a spin one particle and constraining it to have no zero helicity components. This description is free of singularities and we will use it in the following. We choose the scalar product:

$$
(\phi, \psi)=\sum_{i=1}^{3} \int d \mathbf{p} \phi_{i}^{*}(\mathbf{p}) \psi_{i}(\mathbf{p})
$$

The generators of the Poincaré group are unitarily equivalent to

$$
\begin{gathered}
\mathbf{P}=\mathbf{p}, \quad H=\sqrt{\left(\mathbf{p}^{2}\right)} \\
\mathbf{J}=i \frac{\partial}{\partial \mathbf{p}} \wedge \mathbf{p}+\mathbf{s}, \quad \mathbf{K}=\frac{i}{2}\left\{\frac{\partial}{\partial \mathbf{p}}, H\right\}-\frac{\mathbf{s} \wedge \mathbf{p}}{H} .
\end{gathered}
$$

Here $\mathbf{P}, H, \mathbf{J}$ and $\mathbf{K}$ are the generators of space translations, time evolution and boosts, $\mathbf{s}$ are the three dimensional generators of the $S O(3)$ group. The parity and time inversion operators, $\Pi$ and $\Theta$ act on the wave function as follows

$$
\Pi \psi_{i}(\mathbf{p})=\psi_{i}(-\mathbf{p}), \quad \Theta \psi_{i}(\mathbf{p})=\tau_{i j} \psi_{j}^{*}(-\mathbf{p})
$$

The matrix $\tau$ is defined by the equation

$$
\tau^{\dagger} s \tau=-s^{*}
$$

The wave function, in addition, is constrained to have a vanishing zero-helicity component

$$
\frac{(\mathbf{p} . \mathbf{s})^{2}}{\mathbf{p}^{2}} \psi=\psi
$$

The operator $\frac{(\mathbf{p} . \mathbf{s})^{2}}{\mathbf{p}^{2}}$ is the projector onto non vanishing helicity states. note that it commutes with the generators of the Poincaré group, as it should. Now that the photon description is complete we have to define the position operator. We will do this by requiring that it satisfies certain properties and then look for operators that do obey these requirements. 
The position operator eigenvalues must be real so the operator must be self-adjoint

$$
\mathbf{x}=\mathbf{x}^{\dagger}
$$

It must transform under space translations and rotations as a vector

$$
e^{i \mathbf{P} \cdot \mathbf{a}} \mathbf{x} e^{-i \mathbf{P} \cdot \mathbf{a}}=\mathbf{x}+\mathbf{a}, \quad e^{i \mathbf{J} \cdot \mathbf{n}} \mathbf{x}_{i} e^{-i \mathbf{J} \cdot \mathbf{n}}=R(\mathbf{n})_{i}^{j} \mathbf{x}_{j} .
$$

Here $\mathrm{R}(\mathrm{n})$ is the three dimensional rotation matrix. Taking infinitesimal transformations in equations (2.7), we find the commutation relations

$$
\left[x_{i}, p_{j}\right]=i \delta_{i j}, \quad\left[x_{i}, J_{j}\right]=i \epsilon_{i j k} x_{k} .
$$

Lastly we require the position operator to transform as the classical position under parity and time inversion transformations

$$
\Pi \mathbf{x} \Pi=-\mathbf{x}, \quad \Theta \mathbf{x} \Theta^{-1}=\mathbf{x} .
$$

In addition the position operator must act on physical states, those satisfying the constraint (2.5), and the result must also be a physical state, so we have to impose the constraint

$$
\left[\mathbf{x}, \frac{(\mathbf{p} . \mathbf{s})^{2}}{\mathbf{p}^{2}}\right]=0
$$

Equations (2.8) and (2.6) are thus the properties we demand from a position operator. We will show that there exists only one operator satisfying these equations. Equations (2.8a) are solved as follows

$$
\mathbf{x}=i \frac{\partial}{\partial \mathbf{p}}+\mathbf{f}
$$

where $\mathbf{f}$ is a vector constructed out of the momentum and spin operators. There are three basic vectors, $\mathbf{p}, \mathbf{s}$ and $\mathbf{p} \wedge \mathbf{s}$. The operator $\mathbf{f}$ is a linear combination of these vectors with scalar coefficients. The scalars may be constructed out of functions of $\mathbf{p}^{2}$ and of p.s. The latter is actually a pseudoscalar, so it can only be the coefficient of $\mathbf{s}$; this combination does not have the correct behavior under time inversion, so it must be dropped. Note that, due to the constraint (2.5), higher powers of p.s can be dropped too. The same line of argument can be used to eliminate the term proportional to $\mathbf{p}$ so that we are left with the solution to equations $(2.6),(2.8 a)$ and $(2.8 b)$

$$
\mathbf{f}=g\left(\mathbf{p}^{2}\right) \mathbf{p} \wedge \mathbf{s},
$$

where $\mathrm{g}$ is an arbitrary function of $\mathbf{p}^{2}$. In order to have the correct dimension it must be proportionnal to $\frac{1}{\mathbf{p}^{2}}$, the remainig coefficient being determined by the constraint $(2.8 c)$ and equal to 1 . Finally we get the unique solution

$$
\mathbf{x}=i \frac{\partial}{\partial \mathbf{p}}+\frac{\mathbf{p} \wedge \mathbf{s}}{\mathbf{p}^{2}}
$$

This operator has been proposed for the first time by Pryce [6] when looking for the analogue of the centre of mass for the Maxwell equation. It may be written in a representation independent way

$$
\mathbf{x}=\frac{1}{2}\left\{\frac{1}{H}, \mathbf{K}\right\}
$$

The mean value of the Pryce position operator in a state described by the wavefunction $\psi$ can be obtained by the formula

$$
<\mathbf{x}>=\int d \mathbf{r} \hat{\psi}^{\dagger}(\mathbf{r}) \mathbf{r} \hat{\psi}(\mathbf{r})+\frac{i}{4 \pi} \int d \mathbf{R} d \mathbf{u} \hat{\psi}^{\dagger}\left(\mathbf{R}+\frac{\mathbf{u}}{2}\right) \frac{\mathbf{u} \wedge \mathbf{s}}{u^{3}} \hat{\psi}\left(\mathbf{R}-\frac{\mathbf{u}}{2}\right)
$$


where $\hat{\psi}$ if the Fourier transform of $\psi$

$$
\hat{\psi}(\mathbf{r})=\int \frac{d \mathbf{p}}{(2 \pi)^{\frac{3}{2}}} \psi(\mathbf{p}) e^{i \mathbf{p . r}} .
$$

One can thus answer questions such as: given the wave function of the photon what is the mean value of the position observable? The answer includes the usual expected term, the first term on the right hand side of equation (2.13), and an additionnal term, absent for the non-relativistic massive particle.

An important property of the Pryce operator is that it has non-commuting components, the commutator being given by

$$
\left[x_{i}, x_{j}\right]=-i \epsilon_{i j k} p_{k} \frac{\mathbf{p . s}}{p^{3}} .
$$

\section{Optimal localization}

The three components of the Pryce operator cannot be simultaneousely diagonalized, so that one cannot find states localized exactly at a given point in space. Newton and Wigner argued that this makes the photon non-localizable. However, we will show that although the commutator of the three components is non-vanishing, one can find states which are "localised" within an arbitrarily small region.

Before doing so we have to give a meaning to the statement "a photon is localized in a region $(\mathrm{R})$ of space". For simplicity, take the region $(\mathrm{R})$ to be a ball surrounding the origin

$$
\text { (R) } \vec{r}^{2} \leq r_{0}^{2} .
$$

We define a state to be localized in the region $(\mathrm{R})$ if it is a superposition of the eigenstates of the operator $\mathrm{x}^{2}$ with eigenvalues less than or equal to $r_{0}^{2}$. Note that if the components of the position operator were commuting, this definition would agree with the usual one that states that the state must be a superposition of the eigenstates of the position operator with eigenvalues in the region $(\mathrm{R})$.

It turns out that the eigenvalues of the square of the Pryce operator $\mathbf{x}^{2}$ can be arbitrarily small but non-vanishing. In momentum space, the solution to the equation

$$
\mathbf{x}^{2} \psi_{r_{0}}(\mathbf{p})=r_{0}^{2} \psi_{r_{0}}(\mathbf{p})
$$

reads

$$
\psi_{r_{0}}(\mathbf{p})=N \Psi\left(r_{0} \mathbf{p}\right)
$$

where $\mathrm{N}$ is a normalization constant and $\Psi$ is given by

$$
\Psi(\mathbf{u})=\frac{1}{\sqrt{u}} J_{\frac{\sqrt{5}}{2}}(u) E_{ \pm}(\mathbf{u}) v .
$$

$J$ is the Bessel function, $v$ is an arbitrary constant vector and $E_{ \pm}$is the projector onto positive and negative helicity states

$$
E_{ \pm}=\frac{1}{2} \frac{\text { u.s }}{u}\left(1 \pm \frac{\mathbf{u} . \mathbf{s}}{u}\right) .
$$

We conclude that one can find states which arelocalised, in the sense defined above, in the region (R) with the radius $r_{0}$ having an arbitrary non zero value.

Multiplying the function $\Psi$ by $e^{-i \mathbf{p} \cdot \mathbf{x}_{\mathbf{0}}}$ we translate the origin to $\mathbf{x}_{\mathbf{0}}$.

The function $\Psi$ vanishes at the origin. It is roughly speaking, a superposition of momentum eigenstates corresponding to wavelengths smaller than $r_{0}$, from this one can deduce an uncertainty relation, due to the non-commutativity of the Pryce operator components

$$
\Delta x \geq \lambda_{\max },
$$

$\lambda_{\max }$ is the greater wavelength that contributes to the photon wave function.

For photons having a small dispersion in their wavelength, the usual position momentum uncertainty dominates over the one given by the relation (3.6). However, it would be interesting to see whether one can prepare experimentally photon states where the uncertainty due to non-commutativity of the position components dominates over the one due to the non-commutativity of the position and momentum operators. This would constitute a test for the validity of the Pryce operator. 


\section{Summary and conclusion}

We showed that the only self-adjoint operator having the correct transformation properties under translations, rotations, parity and time inversion transformations, and that commutes with the projector over physical states, is the Pryce operator (2.11). We gave the expression of the mean value of the position observable in formula (2.13), and proved that one can find states which are localized in an arbitrarily small region in space. Finally, we argued that one of the experimental tests of the validity of this operator would be the test of the uncertainty relation (3.6) that arises due to the non-commutativity of the components of the Pryce operator . Another test related to the Berry phase has been proposed by Skagerstam [10].

\section{Acknowledgements}

I am grateful to H. Bergeron, T. Brodkorb, S. Caser and R. Omnès for many helpful discussions, and to A. Valance for encouragement.

\section{References}

[ 1] C.K. Hong and L. Mandel, Phys. Rev. Lett. 56 (1986) 58.

[2] T.D. Newton and E.P Wigner, Rev. Mod. Phys. 21 (1949) 400.

[ 3] A.S. Wightman, Rev. Mod. Phys. 34 (1962) 845.

[ 4] J.M. Jauch and C. Piron, Helv. Phys. Acta 40 (1967) 559.

[ 5] W.O. Amrein, Helv. Phys. Acta 42 (1969) 149.

[ 6] M.H.L. Pryce, Proc. Ror. Soc. (London) A195 (1948) 62.

[ 7] H. Bacry, Annls. Inst. Henri Poincaré 49 (1988) 245.

8] E.P. Wigner, Ann. Math. 40 (1939) 149.

[ 9] B.S. Skagerstam and A. Stern, Nucl. Phys. B294 (1987) 636.

[ 10] B.S Skagerstam, preprint ITT 92-09, (1992). 